\title{
Mechanistic Insight of Metalloporphyrin-based Fluorescence Sensor Reacting with Volatile Organic Compounds
}

\author{
Haiyang Gu, ${ }^{*}$ Xingyi Huang, ${ }^{2}$ Quansheng Chen, ${ }^{1,2}$ Yanhui Sun, ${ }^{1}$ and Chin Ping Tan ${ }^{1,3}$ \\ ${ }^{1}$ School of Bio and Food Engineering, Chuzhou University, 239000 Chuzhou, China \\ ${ }^{2}$ School of Food and Biological Engineering, Jiangsu University, 212013 Zhenjiang, China \\ ${ }^{3}$ Faculty of Food Science and Technology, Universiti Putra Malaysia, 43400 Negeri Selangor, Malaysia
}

(Received February 18, 2020; accepted April 2, 2020)

Keywords: fluorescence sensor, volatile organic compounds, density functional theory, theoretical design

The theoretical design of a metalloporphyrin-based fluorescence sensor was developed for the discrimination of volatile organic compounds (VOCs). Molecular models of cobalt tetraphenylporphine (CoTPP) with small VOCs were investigated by the density functional theory (DFT) method at the Becke, 3-parameter, Lee-Yang-Parr (B3LYP) and LAN2DZ level. The relative energies of CoTPP and its complexes were calculated at three spin states (low, intermediate, and high) to obtain their optimized geometry structures. Singlets were found to be the most stable states for CoTPP and its complexes, except for CoTPP-oxygen and CoTPPpropanol at triplets. The binding performance was represented by the binding energy (BE), which showed the following order based on the most stable states: propane (L3) < oxygen $\left(\mathrm{O}_{2}\right)$ $<$ propanol (L2) < hydrogen sulfide $\left(\mathrm{H}_{2} \mathrm{~S}\right)<$ propionaldehyde $(\mathrm{L} 6)<$ nitrogen $\left(\mathrm{N}_{2}\right)<$ butanone (L5) < ethyl acetate (L4) < trimethylamine (L1). We suggest that the CoTPP-based fluorescence sensor is sensitive to trimethylamine and ethyl acetate, and avoids the interference from propane and oxygen.

\section{Introduction}

Volatile organic compounds (VOCs) are one of the most important indicators of food quality attributes obtained from the processing, storage, and consumption of food. ${ }^{(1,2)}$ They indicate the degrees of various chemical, physical, microbiological, and biochemical changes in food. ${ }^{(3-5)}$ Conventionally, the discrimination of VOCs has always been carried out by traditional component-by-component analysis (i.e., gas chromatography). ${ }^{(3,6)}$ This method gives reliable results on food quality and can be applied to the quality control and quality assurance of food and beverages. However, gas chromatography is time-consuming and requires professional operations, such as preprocessing, preconcentration, column separation, detection, and data analysis. Therefore, a rapid and simple detection method is extremely important both to consumers and industry for quality control and assurance.

*Corresponding author: e-mail: guhaiyang51@163.com https://doi.org/10.18494/SAM.2020.2836 
Over the past decades, various sensor-based methods have been developed to detect and discriminate VOCs for the analysis of food quality. The generalized discrimination of VOCs is always related to the sensor-based technology (i.e., electronic nose) ${ }^{(7,8)}$ which adsorbs and detects analytes using a set of heated metal oxides or polymers. This sensor-based method is inspired by the mammalian olfactory and gustatory systems, ${ }^{(9,10)}$ and consists of a series of chemical-sensitive sensors that produce an electrochemical response to discriminate one analyte from another. In comparison with the traditional component-by-component methods (i.e., liquid and gas chromatographies), the advantages of the sensor-based technology are that it is potentially rapid, time-saving, and less expensive. Despite some successes of the electronic nose in the quality control and assurance of food, the discrimination of analytes at low concentrations and the interference of humidity changes are challenges for this traditional sensor-based method. ${ }^{(11,12)}$

According to previous studies, ${ }^{(13,14)}$ the selectivity and sensitivity of sensor-based methods are based on the molecular interactions between sensors and analytes, such as acid-base interactions, $\pi-\pi$ molecular complexation, dipolar and multipolar interactions, hydrogen bonding, bond formation, and last and least, physical adsorption and van der Waals interaction. We present extensions of our colorimetric sensor array and explore its application to the determination of chemical vapors. Here, we present the development of our fluorescent sensor and investigate the design strategy for the detection and discrimination of VOCs. The theoretical design of the fluorescent sensor is related to two fundamental requirements: (1) the fluorescent sensor must have an interaction center to strongly react with analytes and (2) the fluorescent sensor before and after reacting with analytes must produce a strong change in the fluorescence spectrum. These two requirements indicate that the fluorescent sensor must involve stronger chemical interactions (i.e., Lewis and Brønsted acid/base interactions), rather than simply physical adsorption and van der Waals interaction. Metalloporphyrin is an excellent fluorescence sensor with strong sensor analyte interaction and high fluorescence performance before and after reacting with analytes.

Our purpose was then to develop a theoretical design strategy based on the density functional theory (DFT) for the rapid design of a fluorescence sensor.

\section{Computational Methods}

The metalloporphyrin-based fluorescence sensor is represented by a simple cobalt-porphyrin ring with meso-phenyl groups [i.e., cobalt tetraphenylporphine (CoTPP)]. Metalloporphyrin models (CoTPP-L) were constructed with the CoTPP molecule before and after exposure to VOCs, in which L represents the VOCs. According to previous studies, ${ }^{(15,16)}$ various types of alcohols, aldehydes, esters, amines, and ketones are the important indicators of food quality. Hence, trimethylamine (L1), propanol (L2), propane (L3), ethyl acetate (L4), butanone (L5), and propionaldehyde (L6) were selected to represent the common VOCs in food. To avoid any shortcomings, oxygen $\left(\mathrm{O}_{2}\right)$, nitrogen $\left(\mathrm{N}_{2}\right)$, and sulfur dioxide $\left(\mathrm{H}_{2} \mathrm{~S}\right)$ were also considered in this theoretical study. All molecular structures used in this study were obtained from the Cambridge Crystallographic Data Centre (CDCC). The DFT method with Becke, 3-parameter, 
Lee-Yang-Parr (B3LYP) was performed for each model compound. This theoretical method has been proven to be very suitable in the study of similar metalloporphyrin complexes. ${ }^{(17,18)}$ Geometry optimizations of all model compounds were carried out using a basis set called LANL2DZ at three different states in the gas phase. DFT-based methods at this level have been proven to be very efficient for geometry structures, relative energies, binding energies (BE), and charge distributions. ${ }^{(19-21)}$ All theoretical calculations were carried out with the Gaussian16 program suite.

\section{Results and Discussion}

\subsection{Metalloporphyrin-based fluorescence sensor}

Figure 1 shows the schematic of the metalloporphyrin-based fluorescent sensor before and after exposure to VOCs. This fluorescent sensor is dependent on strong and relatively specific interactions (i.e., Lewis acid/base interaction) between sensors and analytes. It is different from the well-known electronic nose approach, which is based on extremely weak and nonspecific interactions (i.e., physical adsorption and van der Waals interaction) between sensors and analytes. ${ }^{(13,22)}$ Therefore, prior weak sensor analyte interaction will provide limited sensitivity and relatively poor selectivity for these traditional methods. Over the past few years, a novel fluorescence sensor method has been developed to provide a unique fluorescence spectrum for the discrimination of VOCs even at low concentrations. The reason for this is that metalloporphyrin-based fluorescence sensors rely on strong molecular interactions between sensors and analytes for molecular recognition. According to previous studies, ${ }^{(13,23)}$ metal-

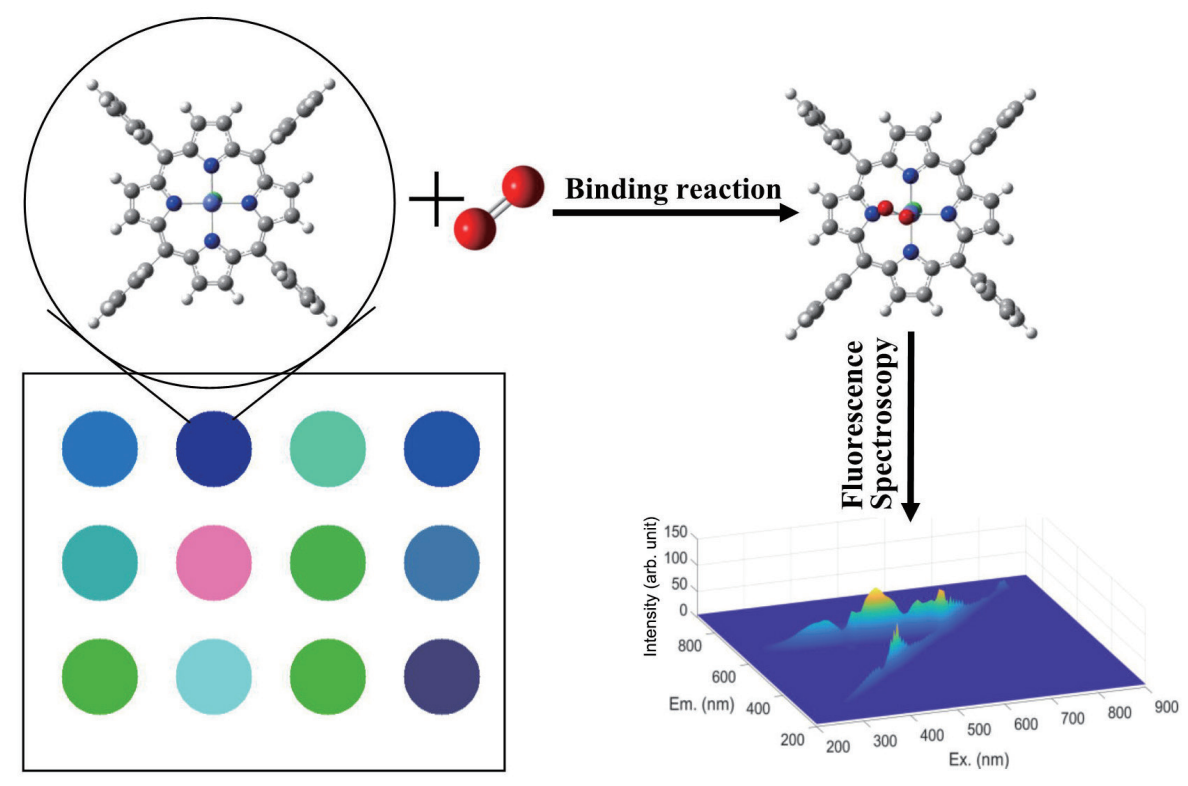

Fig. 1. (Color online) Schematic diagram of metalloporphyrin-based fluorescent sensor interacting with oxygen, similar to all the other metalloporphyrin-VOC systems. 
ligand (i.e., metalloporphyrin-analyte) bonds are intrinsically 20000 -fold more sensitive than these weak interactions (i.e., van der Waals interaction and physical adsorption). Hence, the molecular reaction mechanism between metalloporphyrin-based fluorescence sensors and analytes was needed to develop novel potential applications for food industry and consumers.

\subsection{Binding style of CoTPP-based fluorescence sensor}

The unligated CoTPP model serves as an initial point for discriminating the binding performance of VOCs to the CoTPP molecule. Figure 2 shows the geometry structure of the metalloporphyrin-oxygen model with the labels of different atom types. When the VOC analytes coordinate with the CoTPP-based fluorescence sensor, it is reasonable to expect that the angle and distance (i.e., steric geometry structures) between the VOC analytes and the CoTPP-based fluorescence sensor are important for further calculations. There are two possible binding styles of VOCs, namely, the end-on and side-on binding styles. ${ }^{(24-26)}$ It is generally accepted that the end-on binding style is the most common style of molecular interaction. ${ }^{(27,28)}$ This is also well proved by X-ray structure determination and theoretical calculation, ${ }^{(29)}$ which demonstrates that the calculated energy of the side-on binding style is higher than that of the end-on binding style by about $28 \mathrm{kcal} / \mathrm{mol} .{ }^{(30)}$ Therefore, the end-on bent binding style was selected in this study. Aside from the effect of binding style, molecular distance also has effects on binding performance. According to previous studies, the distance between the metal atom at the center of the metalloporphyrin and VOCs is about 2 to $3 \AA .^{(29,31)}$ Therefore, the effect of VOCs on the binding performance was calculated by adding the VOC molecule at a distance of $2.20 \AA$. These constructed geometry structures of the CoTPP-based fluorescence sensor are expected to be changed by geometry optimization.

\subsection{Relative energies}

To obtain the most stable geometry structures, the geometry structures of the CoTPP-based fluorescence sensor before and after reacting with VOCs were optimized at three different

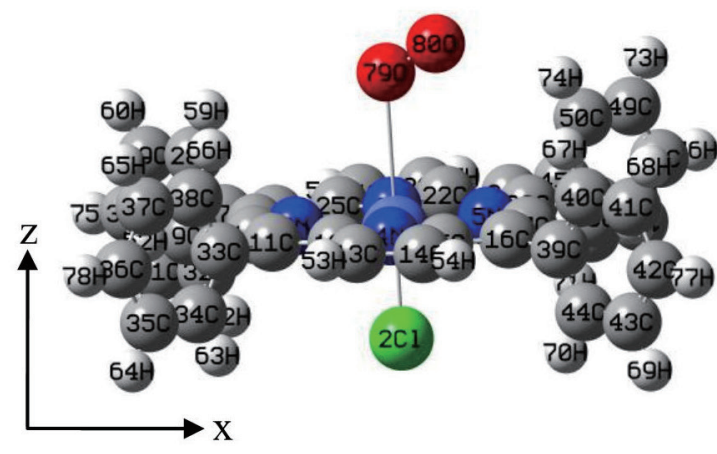

(a)

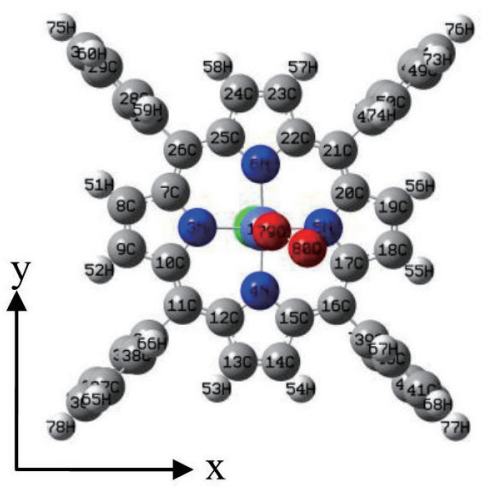

(b)

Fig. 2. (Color online) Optimized geometry structures of CoTPP- $\mathrm{O}_{2}$, similar to all the other CoTPP-VOCs models. The angular Co-O-O structure is shown in the (a) side and (b) top views. 
possible states (i.e., low-spin, intermediate-spin, and high-spin states), as stated previously. ${ }^{(18,20,32)}$ As can be seen in Fig. 3, for the unbound CoTPP model, the singlet state is much more stable than the triplet and quintet states, which is consistent with a previous theoretical study. The relative energies of the optimized triplet and quintet states are 5.72 and $61.82 \mathrm{kcal} / \mathrm{mol}$ larger than that of the singlet state, respectively. For the CoTPP-VOC models, the singlet state is also more stable than the other states, except CoTPP-O 2 and CoTPP-L2 for the triplet state, which is similar to previous studies. ${ }^{(19,20)}$ According to previous reports, ${ }^{(19,33)}$ the smaller energy gap among these three possible states probably causes the binding process to go through more than one reaction pathway, which may make the binding process much more efficient and faster. Note that CoTPP-L4 exhibits a nearly degenerate energy gap, followed by CoTPP, CoTPP- $\mathrm{N}_{2}$, CoTPP- $\mathrm{H}_{2} \mathrm{~S}$, and CoTPP-L1,6, whereas CoTPP-O ${ }_{2}$ and CoTPP-L2,3,5 exhibit larger energy gaps. In the future, this theoretical result must be considered in the design of fluorescence sensor systems for a specific analyte.

\subsection{Molecular structures}

The atomic labels of CoTPP before and after binding different VOCs were applied uniformly in this study, as shown in Fig. 2. According to a previous study, ${ }^{(34)}$ the molecular structures of metalloporphyrin models were mostly located in the $x y$ plane. Therefore, the flatness of the optimized geometry structures could be represented by the $z$-axis coordinated atoms except hydrogen. The planarity and molecular change for CoTPP models before and after binding VOCs were obtained by subtracting the unligated CoTPP structure from the CoTPP-VOC structure. According to the calculated results, the flatness and planarity of the optimized geometry structures were excellent in the metalloporphyrin plane. There was almost no change in the molecular structures of metalloporphyrin before and after exposure to VOCs, such as

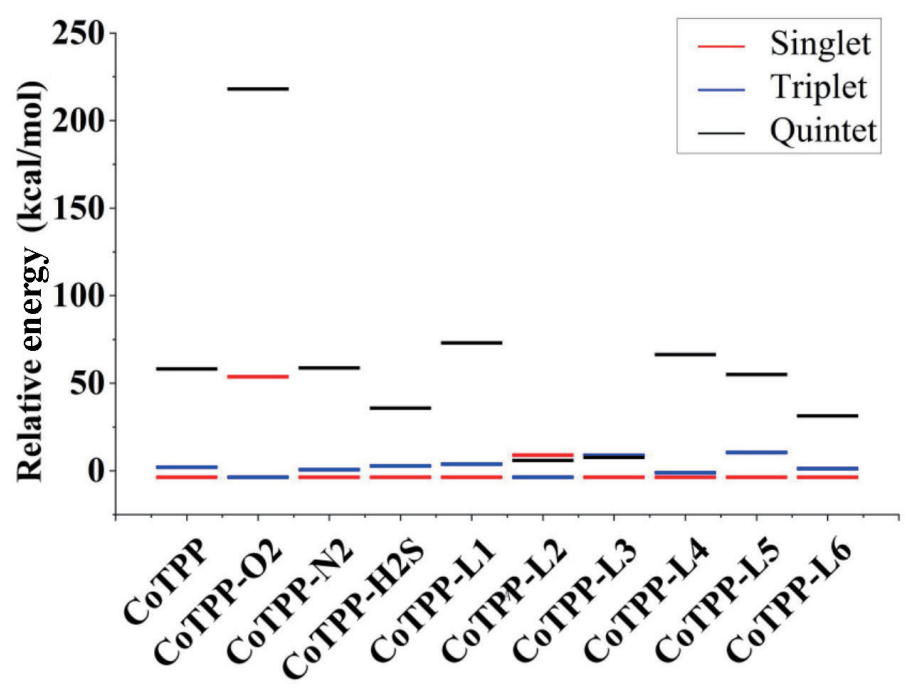

Fig. 3. (Color online) Relative energies of CoTPP-based fluorescence sensor before and after reacting with VOCs based on the most stable one $(\mathrm{kcal} / \mathrm{mol})$. L1 is trimethylamine, L2 is propanol, L3 is propane, L4 is ethyl acetate, L5 is butanone, and L6 is propionaldehyde. 
C11-C12, C12-C13, C10-C11, and C9-C10. However, the molecular structures close to the metal atom at the center of the metalloporphyrin plane showed relatively larger changes than the others, as shown in Fig. 4. The reason for this may be that the Co atoms were pulled out of the metalloporphyrin plane to form a funneled cavity for the facilitated binding of VOCs, which is consistent with a previous report. ${ }^{(19)}$ Note that the angle changes in the metalloporphyrin plane were followed by a number of Co atoms pulled-out of the metalloporphyrin plane. Hence, it is easy to conclude that the molecular interaction between metalloporphyrin and VOCs can cause significant molecular changes, thereby closely leading to the different binding performance characteristics of the VOCs.

\subsection{Frontier molecular orbital energies}

Aside from their effect on the geometry structures, the ligated VOCs also have effects on the frontier molecular orbital energies. The HOMO-1, HOMO, LUMO, and LUMO+1 gaps of the CoTPP-based fluorescence sensor before and after binding VOCs are shown in Fig. 5. The HOMO-LUMO gaps of CoTPP and its complexes were as follows: CoTPP-L2 $(2.047 \mathrm{eV})<$ CoTPP- $\mathrm{H}_{2} \mathrm{~S}(2.422 \mathrm{eV})<$ CoTPP $(2.431 \mathrm{eV})<$ CoTPP-N $2(2.462 \mathrm{eV})<$ CoTPP-L5 $(2.476 \mathrm{eV})<$ CoTPP-L3 $(2.489 \mathrm{eV})<$ CoTPP-L4 $(2.492 \mathrm{eV})<$ CoTPP-L6 (2.506 eV) < CoTPP-L1 (2.604 eV) $<$ CoTPP-O $2(3.038 \mathrm{eV})$, and all the energy gaps were smaller than those shown in a previous report. ${ }^{(35)}$ According to previous studies, ${ }^{(36,37)}$ the smaller energy gaps probably cause the reaction processes to be more efficient, therefore making the binding reaction much more faster and efficient. Note that the increase in energy gap may be due to the decreased HOMO energy levels ( $\sim 0.049$ to $0.395 \mathrm{eV})$ of the calculated models. Additionally, it is generally accepted that HOMO and LUMO serve as the donor and acceptor in the whole model, respectively. The HOMO orbital levels are very similar to each other for the selected CoTPP and its complexes.

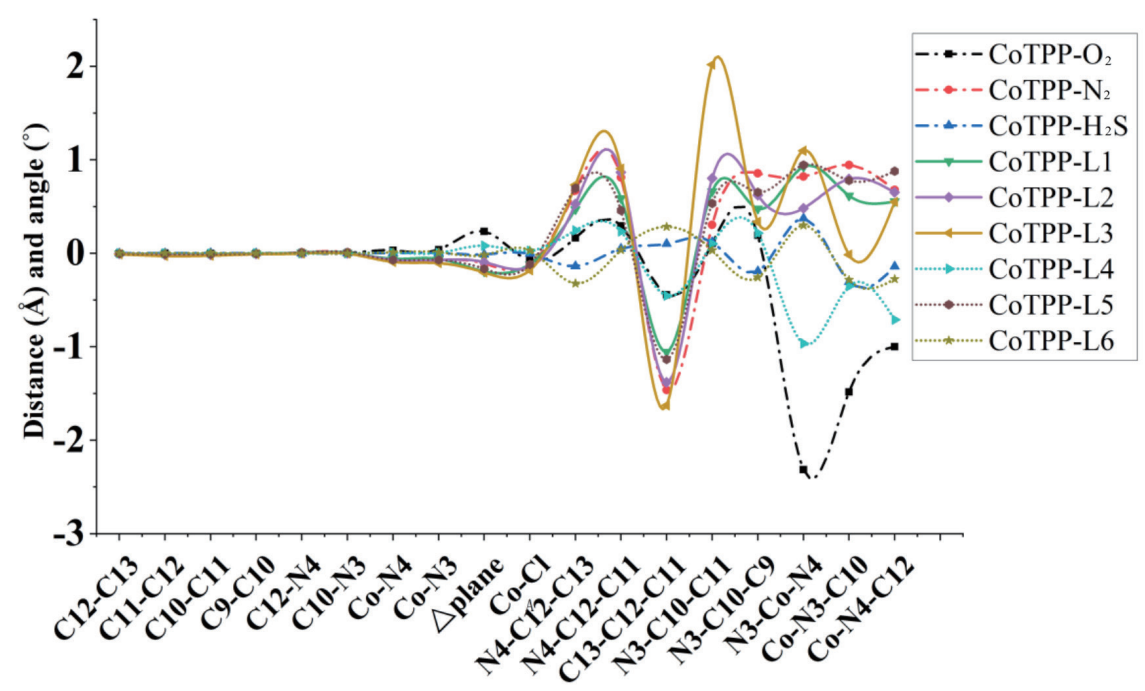

Fig. 4. (Color online) Distances and angles of CoTPP-based fluorescence sensor before and after binding with VOCs. 


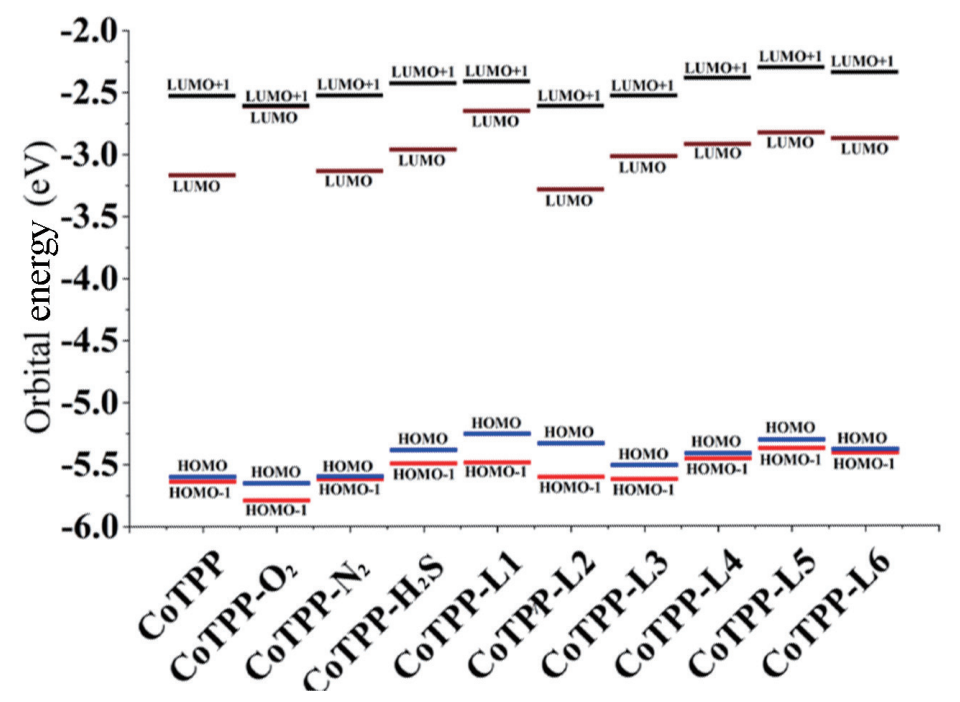

Fig. 5. (Color online) Frontier molecular orbital energies of CoTPP-based fluorescence sensor before and after binding different VOCs.

However, the frontier molecular orbitals of all the calculated models are different, which is consistent with a previous study. ${ }^{(35)}$ The reason for this may be that the different VOCs exhibit electron-withdrawing strengths and affect the electron density in the basic metalloporphyrin plane. Hence, this result reveals that the frontier molecular orbital energies of the CoTPP-based fluorescence sensor can be changed by the addition of suitable VOCs.

\subsection{BE}

The binding performance characteristics of the CoTPP-based fluorescence sensor involve bond formation and breaking, which are closely related to energy changes. To ensure that our energy results were reliable, the electronic and thermal energies were added on the basis of the most stable states. The process of binding the CoTPP-based fluorescence sensor with different VOCs was written as CoTPP + VOCs $\rightarrow$ CoTPP - VOCs. BEs are always used to refer to the energy changes related to this binding process. ${ }^{(19)}$ The BEs obtained by subtracting the energy of the product from the reactants showed more distinct changes on the CoTPPbased fluorescence sensor for each analyte, thus making it more convenient to design sensors for further studies. The $\mathrm{BE}$ can be obtained as $B E=E_{\text {CoTPP }}+E_{V O C S}-E_{\text {CoTPP-VOCs }}$. Figure 6 shows the BEs $(\mathrm{kcal} / \mathrm{mol})$ for all the CoTPPs and their complexes. Note that CoTPP-L1 exhibits the largest BE, followed by CoTPP-N 2 and CoTPP-L4,5,6, whereas CoTPP- $\mathrm{O}_{2}, \mathrm{CoTPP}-\mathrm{H}_{2} \mathrm{~S}$, and CoTPP-L2,3 exhibit smaller BEs. All the calculated results mentioned above indicate that the binding of VOCs can induce significant changes in the optimized geometry structures, frontier molecular orbital energies, and BEs. These changes were closely related to the molecular interaction between CoTPP and VOCs, thereby causing the different performance characteristics for the VOCs. 


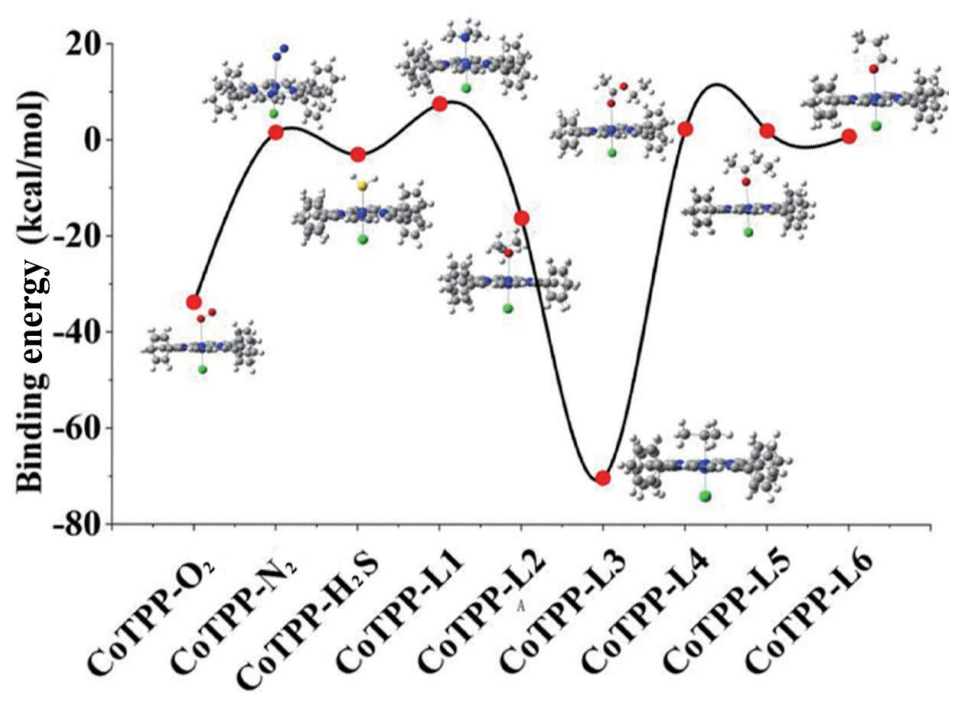

Fig. 6. (Color online) BEs (kcal/mol) related to CoTPP-based fluorescence sensor for different VOCs based on the most stable one.

\section{Conclusions}

The CoTPP-based fluorescence sensor capable of rapidly and simply discriminating VOCs of food was investigated. The calculated models were constructed from the addition of VOCs at appropriate distance and angle above the center of the metal atom. The relative energies of CoTPP and its complexes were calculated to obtain the most stable geometry structures at three different spin states. The BEs of the CoTPP-based fluorescence sensor before and after exposure to the VOCs were calculated as an indicator of sensor performance. All the results mentioned reveal that the CoTPP-based fluorescence sensor is sensitive to trimethylamine and ethyl acetate, and avoids the interference from propane and oxygen. Theoretical results suggest that the CoTPP-based fluorescence sensor is useful for the quality assurance and control of trimethylamine, which is one of the most important indicators of fish freshness. Further research is required to confirm the sensor performance by the application of the sensor in a test.

\section{Acknowledgments}

This study was sponsored by the National Natural Science Foundation of China (Grant nos. 31671932 and 31701685), the Anhui Postdoctoral Science (Chuzhou University) Foundation (Grant no. 2020BSH001), and the Science and Technology Program of Chuzhou (Grant no. 2019ZN006).

\section{References}

1 L. Pillonel, J. Bosset, and R. Tabacchi: LWT-Food Sci. Technol. 35 (2002) 1.

2 A. Loutfi, S. Coradeschi, G. K. Mani, P. Shankar, and J. B. B. Rayappan: J. Food Eng. 144 (2015) 103.

3 P. Perez-Hurtado, E. Palmer, T. Owen, C. Aldcroft, M. Allen, J. Jones, C. S. Creaser, M. R. Lindley, M. A. Turner, and J. C. Reynolds: Rapid Commun. Mass Spectrom. 31 (2017) 1947. 
4 T. Wu, X. Wang, D. Li, and Z. Yi: Atmos. Environ. 44 (2010) 5065.

5 W. Wardencki, M. Michulec, and J. Curyło: Int. J. Food Sci. Technol. 39 (2004) 703.

6 Y. Nolvachai, C. Kulsing, and P. J. Marriott: TrAC, Trends Anal. Chem. 96 (2017) 124.

7 H. G. J. Voss, J. J. A. Mendes Júnior, M. E. Farinelli, and S. L. Stevan: Sensors 19 (2019) 2646.

8 H. Li, D. Luo, Y. Sun, and H. GholamHosseini: Sens. 19 (2019) 5033.

9 P. Mombaerts: Nat. Rev. Neurosci. 5 (2004) 263.

10 H. Matsunami, J.-P. Montmayeur, and L. B. Buck: Nat. 404 (2000) 601.

11 Y. Zheng, H. Li, W. Shen, and J. Jian: Sens. Actuators A: Phys. 285 (2019) 395.

12 C. Sun and Y. Cao: Int. Conf. Applications and Techniques in Cyber Security and Intelligence. 1 (2019) 281.

13 K. S. Suslick, N. A. Rakow, and A. Sen: Tetrahedron 60 (2004) 11133.

14 M. C. Janzen, J. B. Ponder, D. P. Bailey, C. K. Ingison, and K. S. Suslick: Anal. Chem. 78 (2006) 3591.

15 L. Oro, E. Feliziani, M. Ciani, G. Romanazzi, and F. Comitini: Int. J. Food Microbiol. 265 (2018) 18.

16 V. Capozzi, S. Makhoul, E. Aprea, A. Romano, L. Cappellin, A. Sanchez Jimena, G. Spano, F. Gasperi, M. Scampicchio, and F. Biasioli: Mol. 21 (2016) 483.

17 L. J. He, Y. Sun, W. Li, J. Wang, M. X. Song, and H. X. Zhang: Solar Energy 173 (2018) 283.

18 H. d. L. B. Cavalcanti, and G. B. Rocha: J. Mol. Model. 23 (2017) 363.

19 Y. Sun, X. Hu, H. Li, and A. F. Jalbout: J. Phys. Chem. C. 113 (2009) 14316.

20 A. Abdurahman and T. Renger: J. Phys. Chem. A. 113 (2009) 9202.

21 I. M. Nielsen and K. Leung: The J. Phys. Chem. A. 114 (2010) 10166.

22 C. Zhang and K. S. Suslick: J. Agric. Food Chem. 55 (2007) 237.

23 K. S. Suslick, N. A. Rakow, M. E. Kosal, W. B. McNamara III, and A. Sen: Proc. Int Soc. Olfaction Electron Noses 1 (2003) 46.

24 H. Gu, H. Shi, Y. Sun, and H. Xu: Mater. Express 8 (2018) 93.

25 H. Gu, Y. Sun, S. Li, X. Huang, and H. Dai: Comput. Theor. Chem. 1094 (2016) 13.

26 C. Chen, C. Tang, W. Xu, Y. Li, and L. Xu: Phys. Chem. Chem. Phys. 20 (2018) 9536.

27 L. L. Zhang, X. Y. Wang, K. Y. Jiang, B. Y. Zhao, H. M. Yan, X. Y. Zhang, Z. X. Zhang, Z. Guo, and C. M. Che: Dalton Trans. 47 (2018) 5286

28 V. H. A. Pinto, N. K. Falcão, J. C. Bueno-Janice, I. Spasojević, I. Batinić-Haberle, and J. S. Rebouças: In Redox-Active Therapeutics. 1 (2016) 213.

29 Y. Sun, X. Hu, H. Li, and A. F. Jalbout: J. Phys. Chem. C. 113 (2009) 14316.

30 H. Dube, B. Kasumaj, C. Calle, M. Saito, G. Jeschke, and F. O. Diederich: Angewandte Chemie. 47 (2010) 2600 .

31 A. Abdurahman and T. Renger: J. Phys. Chem. A. 113 (2009) 9202.

32 M. X. Jiang and C. G. Liu: J. Mol. Graphics Modell. 73 (2017) 8.

33 K. P. Jensen and U. Ryde: J. Biol. Chem. 279 (2004) 14561.

34 L. Wan, D. Qi, and Y. Zhang: J. Mol. Graphics Modell. 30 (2011) 15.

35 N. Santhanamoorthi, C. M. Lo, and J. C. Jiang: J. Phys. Chem. Lett. 4 (2013) 524.

36 K. W. Jolley, P. Wagner, K. Wagner, P. J. Walsh, K. C. Gordon, W. M. Campbell, L. Schmidt-Mende, M. K. Nazeeruddin, Q. Wang, and M. Gratzel: J. Phys. Chem. C. 111 (2007) 11760.

37 P. Guo, H. Cui, X. Zhang, M. K. Nazeeruddin, M. Gratzel, and R. Ma: J. Phys. Chem. 113 (2009) 10119. 